\title{
GROTHENDIECK-RIEMANN-ROCH FOR COMPLEX MANIFOLDS
}

BY NIGEL R. O'BRIAN, DOMINGO TOLEDO ${ }^{1}$ AND YUE LIN L. TONG ${ }^{1}$

For a coherent analytic sheaf $F$ on a complex manifold $X$ and a holomorphic map $f: X \rightarrow Y$ to a complex manifold $Y$, with $f$ proper on the support of $F$, we prove a Grothendieck-Riemann-Roch formula as in [3] relating the Todd classes of $X, Y$ and the Chern character of $I$ and its direct images. This is the first example of a Riemann-Roch theorem for general mappings of complex manifolds which are not necessarily projective varieties. For the history of this problem see $[2,7,8]$.

The theorem of Grauert shows that the direct image sheaves $R^{i} f_{*} F$ are coherent and we prove the formula

$$
\sum_{i}(-1)^{i} \operatorname{ch}\left(R^{i} f_{*} F\right) \operatorname{Todd}(Y)=f_{*}(\operatorname{ch}(F) \operatorname{Todd}(X)) .
$$

The characteristic classes of $X$ and $Y$ are defined as in [1] and the Chern characters as in [9], so that all classes lie in Hodge cohomology. The theorem thus relates analytic, rather than topological, invariants of $F$ and its direct images. The same general methods should also lead to the corresponding statements for topological invariants in singular cohomology [2] and perhaps other cohomology theories. The de Rham Chern classes defined in [6] should be relevant in this context.

The proof is based throughout on the techniques of twisting cochains and local formulae introduced in [12] and developed in subsequent papers, and follows Grothendieck's approach to the extent that we factor $f$ as $\pi^{\circ} \Gamma$ where $\Gamma$ : $X \rightarrow X \times Y$ is the graph of $f$ and $\pi: X \times Y \rightarrow Y$ is the projection. We first prove the following two special cases.

Theorem A. Suppose : $X \rightarrow Z$ is a closed embedding with the property that there exists a holomorphic retraction $\rho: Z \rightarrow X$ of maximal rank in a neighbourhood of $\iota(X)$. Then

$$
\operatorname{ch}\left(\iota_{*} F\right)=\iota_{*}\left(\operatorname{Todd}(N)^{-1} \operatorname{ch}(F)\right)
$$

where $N$ is the normal bundle of $X$ in $Z$.

Received by the editors March 7, 1981.

1980 Mathematics Subject Classification. Primary 14C20, 32L10.

( $\left.{ }^{1}\right)$ Partially supported by NSF Grants MCS 79-02753 and MCS 79-03798. 
THEOREM B. Let $F$ be a coherent sheaf on $X \times Y$ with $\pi \mid$ Supp $F$ proper. Then

$$
\sum_{i}(-1)^{i} \operatorname{ch}\left(R^{i} \pi_{*} F\right)=\int_{X} \operatorname{Todd}(X) \operatorname{ch}(F) .
$$

The fibre integration makes sense because of the support condition, and gives the Gysin map in this case.

The general formula now follows by applying Theorem A with $Z=X \times Y$, $\iota=\Gamma$ and retraction $\rho(x, y)=(x, f(x))$. Theorem $\mathrm{B}$ is then applied with $F$ replaced by $\Gamma_{*} F$.

The proof of Theorem A is straightforward and is based on the theory of the Gysin map of [13] and a version of the trace map of [9]. We construct an explicit twisted resolution of $\iota_{*} F$ which gives a local formula for $\operatorname{ch}\left(\iota_{*} F\right)$, which is then computed explicitly by reducing to the embedding of $X$ in $N$.

The heart of the matter lies in the proof of Theorem B. Locally with respect to $Y$ this is essentially the same as the result of [10], the main difference being that the finiteness and duality theorems and the Künneth formula are replaced by the corresponding relative versions. The finiteness result, proved as in [4] for example, is the local existence of a complex of free $O_{Y}$-modules whose cohomology gives the direct image sheaves. The duality theorem is given in [11] and the Künneth formula also follows from the homological properties of topological vector spaces and completed tensor products.

The principal device used to make these results global on $Y$ is the 'shuffle map' of Eilenberg-Mac Lane [5]. For Stein covers $U, V$ of $X$ and $Y$ and a twisting cochain $(U \times V, F, b)$ for $F$ on $X \times Y$ the shuffle map $\phi$ applied to the cochain $b$ on the product cover gives an 'infinite-dimensional twisting cochain' $(U, \mathbf{F}, \phi(b))$ on $Y$. The local complexes $\mathbf{F}_{\alpha}$ of this twisting cochain are complexes of free nuclear Fréchet $O_{Y}$-modules in the sense of [4] and correspond to the part of the cochain complex $C_{b}(U \times V, F)$ lying over a fixed $V_{\alpha}$ in $V$. This twisting cochain resolves the global complex $C^{\circ}$ of $O_{Y}$-modules with $C^{\circ}(V)$ $=C^{\cdot}(U \times V, F)$ whose cohomology gives the direct image sheaves. This also supplies a natural Atiyah class for $C^{*}$ which is the image of the $Y$-derivative part of the Atiyah class of $F$ under the shuffle map.

The theory of the Gysin map applied as in [10] to the diagonal embedding of $X \times Y$ into $X \times X \times Y$ then gives a formula

$$
\operatorname{ch}\left(C^{\circ}\right)=\int_{X} \operatorname{Todd}(X) \operatorname{ch}(F)
$$

where $\operatorname{ch}\left(C^{\circ}\right)$ is calculated using the powers of the Atiyah class and a trace map obtained by using the Gysin map to represent the resulting cocycles as cocycles of 'smoothing operators' between the local complexes $\mathbf{F}_{\alpha}^{\cdot}$. The proof of this 
formula uses arguments similar to those applied in [10] to identify the restriction of the Lefschetz class to the diagonal.

Theorem B then follows from the relation

$$
\operatorname{ch}\left(C^{\cdot}\right)=\sum_{i}(-1)^{i} \operatorname{ch}\left(R^{i} \pi_{*} F\right)
$$

To prove this we develop a theory of twisting cochains and Chern character for complexes of sheaves, such as $C^{\cdot}$, which are 'perfect' in the sense of [7], i.e., are locally quasi-isomorphic to free finite complexes. For this definition of Chern character the formula above follows easily and so it remains to show that the two definitions agree. This can be reduced to the local Künneth formula and duality argument referred to above.

Other approaches to Theorem B are possible, but the one sketched is the simplest we have found.

\section{REFERENCES}

1. M. F. Atiyah, Complex analytic connections in fibre bundles, Trans. Amer. Math. Soc. 85 (1957), $181-207$.

2. M. F. Atiyah and F. Hirzebruch, The Riemann-Roch theorem for analytic embeddings, Topology 1 (1962), 151-166.

3. A. Borel and J.-P. Serre, Le Théorème de Riemann-Roch, Bull. Soc. Math. France 86 (1958), 97-136.

4. A. Douady, Le théorème des images directes de Grauert (d'après Kiehl-Verdier), Seminaire Bourbaki, exposé 404, (1971).

5. S. Eilenberg and S. Mac Lane, On the groups $H(\Pi, n)$. I, Ann. of Math. (2) 58 (1953), 55-106.

6. H. Green, Preprint, Univ. of Warwick, 1979.

7. A. Grothendieck, Problèmes ouverts en théorie des intersections, Théorie des Intersections et Théorème de Riemann-Roch (SGA 6), Lecture Notes in Math., vol. 225, SpringerVerlag, Berlin and New York, 1971, pp. 667-688.

8. F. Hirzebruch, Komplexe Mannigfaltigkeiten, Proc. Internat. Congr. Math. (1958), Cambridge Univ. Press, New York, 1960, pp. 119-136.

9. N. R. O'Brian, D. Toledo and Y. L. L. Tong, $A$ trace map and characteristic classes for coherent sheaves, Amer. J. Math. 103 (1981), 225-252.

10. - Hirzebruch-Riemann-Roch for coherent sheaves, Amer. J. Math. 103 (1981), 253-271.

11. J.-P. Ramis and G. Ruget, Résidus et dualité, Invent. Math. 26 (1974), 89-131.

12. D. Toledo and Y. L. L. Tong, A parametrix for $\bar{\partial}$ and Riemann-Roch in Čech Theory, Topology 15 (1976), 273-301.

13. - Duality and intersection theory in complex manifolds. I, Math. Ann. 237 (1978), 41-77.

INSTITUTE OF ADVANCED STUDIES; AUSTRALIAN NATIONAL UNIVERSITY, CANBERRA, ACT 2600, AUSTRALIA

DEPARTMENT OF MATHEMATICS, UNIVERSITY OF UTAH, SALT LAKE CITY, DEPARTMENT OF MATHEMATICS, PURDUE UNIVERSITY, WEST LAFAYETTE, INDIANA 47907 